\title{
Intraductal papillary-mucinous carcinoma with portal vein tumor thrombus and multifocal liver metastasis: An autopsy case
}

\author{
NAOHIRO MATSUSHITA ${ }^{1}$, AKITOSHI DOUHARA ${ }^{2}$, HIROTSUGU UENO $^{2}$, SHOHEI ASADA $^{2}$, \\ KOJI MURATA ${ }^{2}$, KOJI YANASE ${ }^{2}$ and MASAHIRO TSUTSUMI ${ }^{3}$ \\ ${ }^{1}$ Clinical Study and Training Center; Departments of ${ }^{2}$ Gastroenterology and ${ }^{3}$ Pathology, \\ Saiseikai Chuwa Hospital, Sakurai, Nara 633-0054, Japan
}

Received May 22, 2020; Accepted February 5, 2021

DOI: $10.3892 / \operatorname{mco} .2021 .2263$

\begin{abstract}
The prognosis of intraductal papillary-mucinous neoplasm is superior to that of conventional pancreatic ductal adenocarcinoma. Only a few advanced cases of intraductal papillary-mucinous carcinoma (IPMC) have been reported to date. We herein report the case of a 78-year-old male patient with advanced pancreatobiliary type IPMC with portal vein invasion and liver metastasis. The IPMC invaded the portal vein to form a tumor thrombus and it also metastasized to the liver via the portal vein. After receiving best supportive care, the patient succumbed to the disease following an exacerbation of IPMC 90 days after the initial presentation. On autopsy, a very long tumor thrombus was identified, along with liver metastatic lesions, which had retained the structure of the primary IPMC on histological examination.
\end{abstract}

\section{Introduction}

Intraductal papillary-mucinous neoplasm (IPMN) of the pancreas is a cystic tumor arising from the cells lining the pancreatic ducts. IPMN is divided into main-duct, branch-duct and mxixed-type lesions, depending on the site of origin (1). The disease spectrum ranges from low-grade dysplasia to invasive intraductal papillary-mucinous carcinoma (IPMC) (2). Moreover, IPMN may occasionally be associated with pancreatic carcinoma at a site distant from IPMN (1). Histologically,

Correspondence to: Dr Akitoshi Douhara, Department of Gastroenterology, Saiseikai Chuwa Hospital, 323 Abe, Sakurai, Nara 633-0054, Japan

E-mail: aki-do@hotmail.co.jp

Abbreviations: IPMC, intraductal papillary-mucinous carcinoma; IPMN, intraductal papillary-mucinous neoplasm; CT, computed tomography; SMV, superior mesenteric vein; EBS, endoscopic biliary stenting; MUC, mucin core protein; PDAC, pancreatic ductal adenocarcinoma

Key words: intraductal papillary-mucinous carcinoma, portal invasion, portal vein tumor thrombosis, liver metastasis
IPMNs are subdivided into intestinal, pancreatobiliary, gastric and oncocytic types based on the expression of immunohistochemical markers, such as mucin core protein (MUC)1, MUC2 and CDX2 (3). Generally, the prognosis following surgical resection of IPMN with an associated invasive carcinoma has been reported to be superior to that of pancreatic ductal adenocarcinoma (PDAC) (4). However, the prognosis of the pancreatobiliary type IPMN is worse compared with that of the other subtypes (5). We herein report a case of a pancreatobiliary type IPMC with formation of a portal vein tumor thrombus and multifocal liver metastasis.

\section{Case report}

A 78-year-old man visited a regional hospital with complaints of fever and vomiting. An abdominal plain computed tomography $(\mathrm{CT})$ scan at the hospital revealed dilatation of the bile ducts and the presence of cystic lesions in the pancreatic head and liver, and the patient was referred to Saiseikai Chuwa Hospital (Sakurai, Japan) for further examination and treatment.

The patient had a history of hypertension and benign prostatic hyperplasia and received regular treatment with nifedipine and tamsulosin hydrochloride. There was no history of liver dysfunction or a specific family history. The patient reported no allergies, cigarette smoking, or alcohol consumption.

When the patient first visited our hospital in March 2018, his vital signs were stable, with a body temperature of $36.3^{\circ} \mathrm{C}$, heart rate of $56 \mathrm{bpm}$, blood pressure of 120/66 $\mathrm{mmHg}$, respiratory rate of 16 breaths/min and $\mathrm{SpO}_{2}$ of $96 \%$ in room air. The conjunctivas were slightly anemic. There were no murmurs or rales on chest auscultation, and the abdomen was soft and flat with no tenderness on palpation. The laboratory tests (Table I) revealed mild normocytic anemia, reduced levels of albumin and increased levels of hepatic and biliary enzymes, including aspartate aminotransferase, alanine aminotransferase, alkaline phosphatase, $\gamma$-glutamyl transpeptidase and pancreatic amylase. The levels of tumor markers, including carbohydrate antigen (CA)19-9, DUPAN-2, carcinoembryonic antigen and $\alpha$-fetoprotein were measured, and the CA19-9 level was found to be increased. To evaluate the pancreatic and liver masses, an abdominal contrast-enhanced CT scan was performed (Fig. 1). A polycystic low-density area was identified in the pancreatic 
Table I. Laboratory data at the patient's first visit.

\begin{tabular}{|c|c|c|}
\hline Laboratory parameters (normal range) & Value & Unit \\
\hline \multicolumn{3}{|l|}{ Hematology } \\
\hline White blood cell count $(3,300-8,600)$ & 4,600 & $/ \mu 1$ \\
\hline Red blood cell count $\left(435-555 \times 10^{4}\right)$ & $351 \times 10^{4}$ & $/ \mu 1$ \\
\hline Hematocrit (40.7-50.1) & 32.1 & $\%$ \\
\hline Hemoglobin (13.7-16.8) & 10.6 & $\mathrm{~g} / \mathrm{dl}$ \\
\hline Mean corpuscular volume (83.6-98.2) & 91.5 & fl \\
\hline Platelet count $\left(15.8-34.8 \times 10^{4}\right)$ & $16.1 \times 10^{4}$ & $/ \mu 1$ \\
\hline \multicolumn{3}{|l|}{ Coagulation } \\
\hline Prothrombin time-INR (0.85-1.15) & 1.03 & INR \\
\hline Activated partial thromboplastin time (25-40) & 31.9 & sec \\
\hline Fibrinogen (150-400) & 491 & $\mathrm{mg} / \mathrm{dl}$ \\
\hline Fibrin degradation products $(0-10)$ & 3.4 & $\mu \mathrm{g} / \mathrm{ml}$ \\
\hline D-dimer $(0-1)$ & 1.0 & $\mu \mathrm{g} / \mathrm{ml}$ \\
\hline \multicolumn{3}{|l|}{ Chemistry } \\
\hline Albumin $(4.1-5.1)$ & 3.2 & $\mathrm{~g} / \mathrm{dl}$ \\
\hline Aspartate transaminase $(13-30)$ & 41 & IU/1 \\
\hline Alanine transaminase $(10-42)$ & 66 & IU/1 \\
\hline Lactate dehydrogenase (124-222) & 86 & IU/1 \\
\hline Alkaline phosphatase (106-322) & 788 & IU/l \\
\hline$\gamma$-Glutamyl transpeptidase (13-64) & 160 & IU/1 \\
\hline Amylase (44-132) & 357 & IU/l \\
\hline P-amylase (16-52) & 282 & IU/1 \\
\hline Total bilirubin (0.4-1.5) & 0.5 & $\mathrm{mg} / \mathrm{dl}$ \\
\hline Blood urea nitrogen $(8-20)$ & 16.5 & $\mathrm{mg} / \mathrm{dl}$ \\
\hline Creatinine (0.65-1.07) & 0.75 & $\mathrm{mg} / \mathrm{dl}$ \\
\hline $\mathrm{Na}(138-145)$ & 134 & $\mathrm{mEq} / \mathrm{l}$ \\
\hline K (3.6-4.8) & 4.2 & $\mathrm{mEq} / \mathrm{l}$ \\
\hline C-reactive protein $(0-0.29)$ & 5.165 & $\mathrm{mg} / \mathrm{dl}$ \\
\hline Procalcitonin $(0-0.4)$ & 0.39 & $\mathrm{ng} / \mathrm{ml}$ \\
\hline Brain natriuretic peptide (0-18.4) & 19.6 & $\mathrm{pg} / \mathrm{ml}$ \\
\hline Hemoglobin A1c (4.7-6.2) & 8.7 & $\%$ \\
\hline \multicolumn{3}{|l|}{ Tumor markers } \\
\hline Carbohydrate antigen $19-9(0-37)$ & 84.6 & $\mathrm{U} / \mathrm{ml}$ \\
\hline DUPAN-2 (0-150) & 46 & $\mathrm{U} / \mathrm{ml}$ \\
\hline Carcinoembryonic antigen (0-5) & 3.5 & $\mathrm{ng} / \mathrm{ml}$ \\
\hline$\alpha$-Fetoprotein $(0-15)$ & 2.0 & $\mathrm{ng} / \mathrm{ml}$ \\
\hline Protein induced by vitamin K absence-II (0-39) & 25.45 & $\mathrm{mAU} / \mathrm{ml}$ \\
\hline
\end{tabular}

INR, international normalized ratio.

head, with a width of $46 \mathrm{~mm}$, and the wall of this polycystic area was partially well-enhanced. The common bile duct was obstructed due to the polycystic tumor in the pancreatic head and was dilated to $20 \mathrm{~mm}$. Multiple polycystic low-density areas were identified in the liver, with a morphology similar to that of the cystic lesion in the pancreatic head. Moreover, a 10-mm filling defect was observed in the superior mesenteric vein (SMV). The magnetic resonance cholangiopancreatography signals from a $\mathrm{T} 2$-weighted image revealed that the pancreatic head tumor exhibited low intensity, with a small high-intensity area (Fig. 2). Similarly, on the CT scan, the common bile duct was obstructed by the polycystic tumor in the pancreatic head and was dilated to a width of $20 \mathrm{~mm}$. In order to drain the bile duct and perform diagnostic brush cytology, endoscopic retrograde cholangiopancreatography (ERCP) was performed (Fig. 3). The papilla of Vater was normal. First, contrast medium was injected into the pancreatic duct. The main pancreatic duct was dilated to a width of $4 \mathrm{~mm}$. Pooling of mucus in the pancreatic duct was suspected due to the shallow filling defects. Second, contrast medium was injected into the bile duct. The proximal bile duct was markedly dilated, whereas the distal bile duct was narrowed 

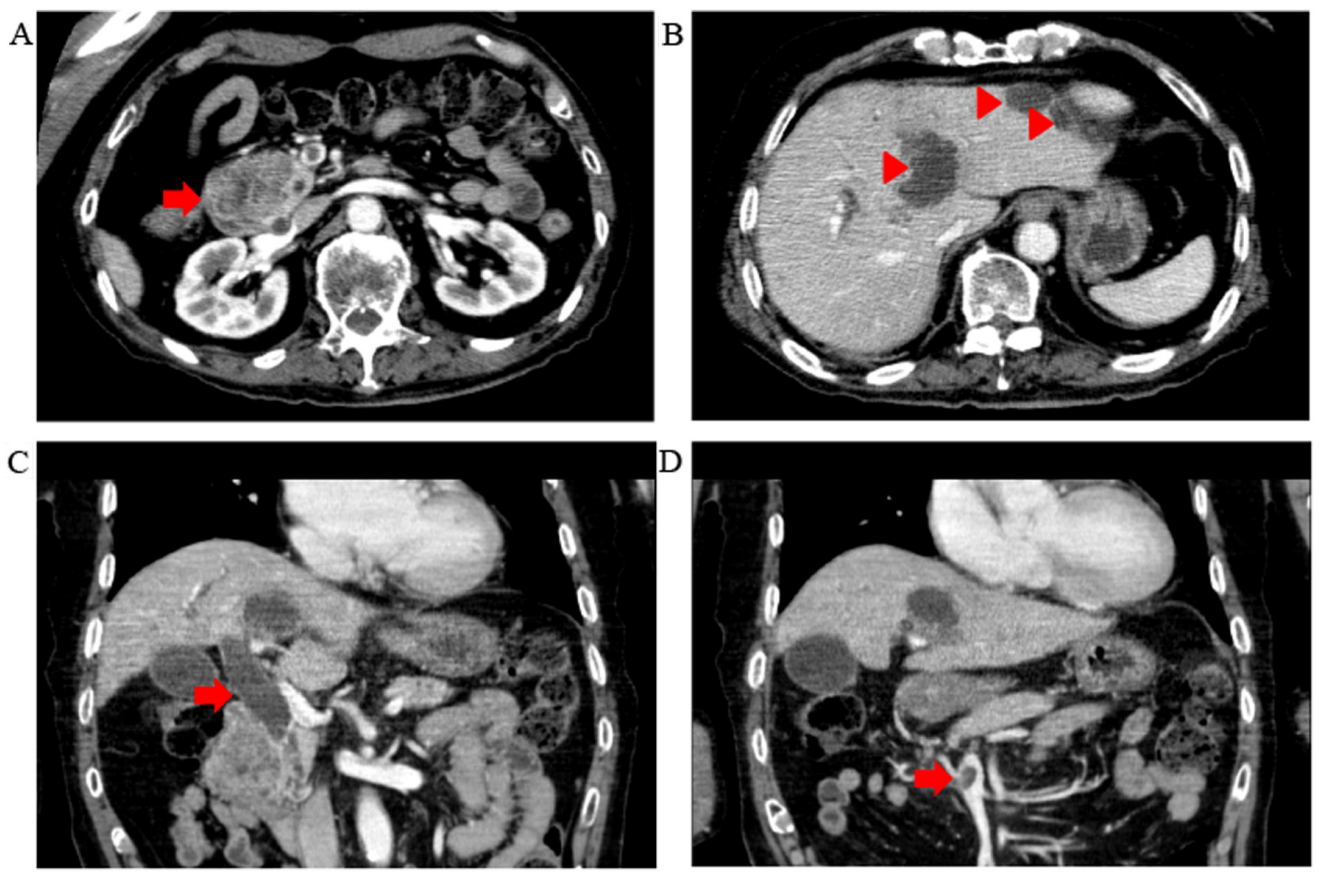

Figure 1. Abdominal contrast-enhanced CT at the patient's first visit. (A) A polycystic tumor was identified in the pancreatic head (arrow). (B) Polycystic low-density areas in the liver (arrowheads). (C) The pancreatic tumor obstructed the common bile duct, and the proximal part of the bile duct was dilated to $20 \mathrm{~mm}$ (arrow). (D) A 10-mm area with lack of enhancement was identified in the superior mesenteric vein (arrow).

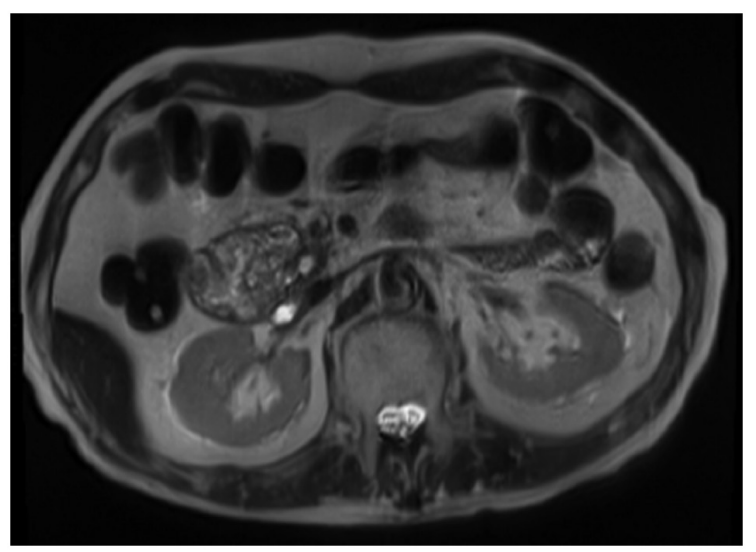

Figure 2. Magnetic resonance cholangiopancreatography signal from a T2-weighted image at the patient's first visit. The pancreatic head tumor exhibited low intensity, with a small high-intensity area.

and was $20 \mathrm{~mm}$ in length. Brush cytology of the narrowed bile duct was conducted, and endoscopic biliary stenting (EBS) was performed using a plastic stent in order to drain the bile duct. The brush cytology findings revealed the presence of atypical ductal cells.

The comprehensive diagnosis was advanced IPMC with invasion of the bile duct and liver metastasis. The clinical stage was stage IV (T3N0M1) according to the Union for International Cancer Control TNM classification (6). As regards treatment, systemic chemotherapy was recommended; however, the patient and his family opted for best supportive care due to his declining performance status (performance status score: 4). Thus, EBS was performed to drain the bile duct on the 2nd day of hospitalization and the plastic stent was exchanged for a metallic stent on the 8th day to prolong stent patency. Subsequently, the patient developed cholangitis and aspiration pneumonia, and he eventually succumbed to IPMC 90 days after the initial admission.

After obtaining informed consent from the patient's daughter, an autopsy was performed to examine the tumor. Macroscopically, the polycystic tumor in the pancreatic head was sized $5 \times 4.5 \mathrm{~cm}$ and the cysts contained mucus. Moreover, the tumor had invaded the portal vein and formed an elongated tumor thrombus, extending from the SMV to the hilar portal vein. Polycystic tumor lesions were also identified in the liver, and the structure of these metastatic lesions was identical to that of the primary pancreatic head tumor (Fig. 4).

Microscopically, on hematoxylin and eosin staining, the pancreatic head tumor exhibited a papilliform structure consisting of small tumor cells and the tumor cysts were filled with mucus. On immunostaining, MUC1 and MUC5AC were positive (Fig. 5), but MUC2 was negative (data not shown). Therefore, the histological diagnosis was pancreatobiliary type IPMC, and the morphological diagnosis, which was based on the dilation of both the main pancreatic duct as well as its branches, was mixed-type IPMC. The polycystic liver tumors and SMV tumor thrombus had the same morphology as the pancreatic tumors on pathological examination, with pooling of mucus in the cysts of the liver metastatic lesions (Fig. 6). The pancreatic head tumor had not metastasized to the regional lymph nodes, but rather directly metastasized to the liver via the portal vein, retaining the IPMC structure and mucus production.

\section{Discussion}

There were two clinical issues with the present case: First, the pancreatic tumor directly invaded the portal vein and caused 

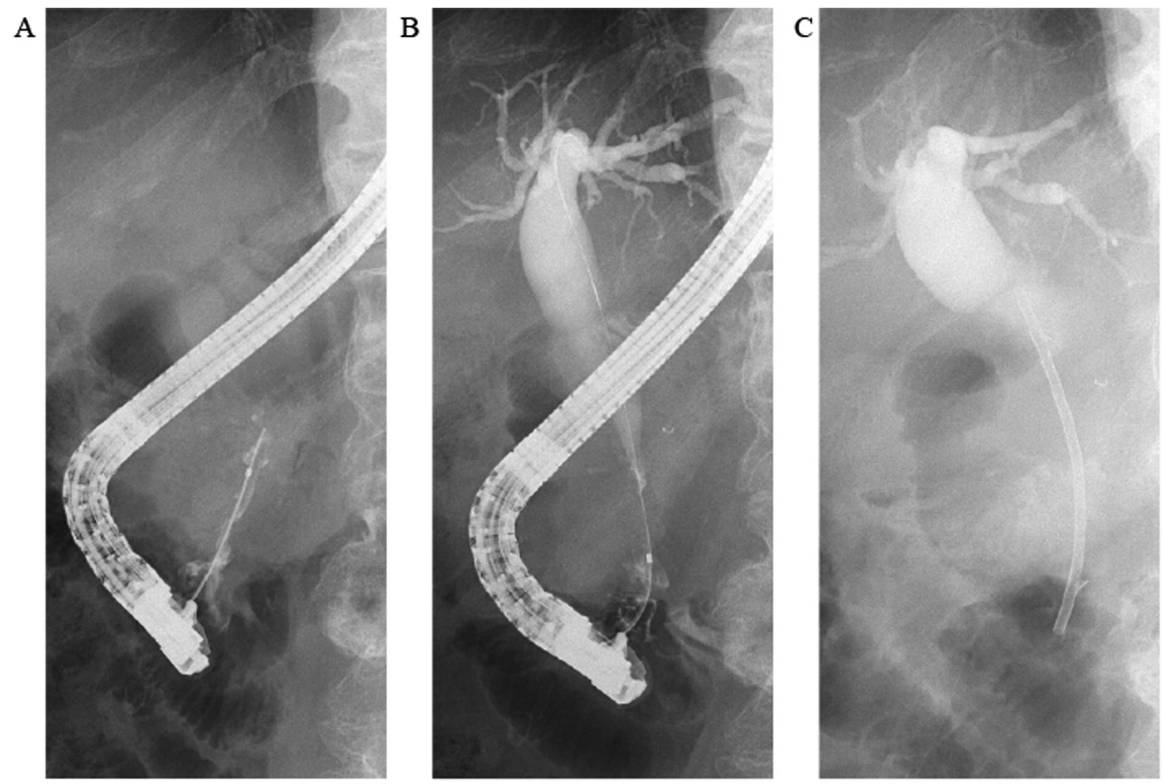

Figure 3. Endoscopic retrograde cholangiopancreatography was performed to drain his bile duct and enable diagnosis. (A) The main pancreatic duct was widened to $4 \mathrm{~mm}$ with mucus pooling. (B) The proximal bile duct was clearly widened, and the distal bile duct was narrowed over a length of $20 \mathrm{~mm}$. (C) Endoscopic biliary stenting was performed using a plastic stent.
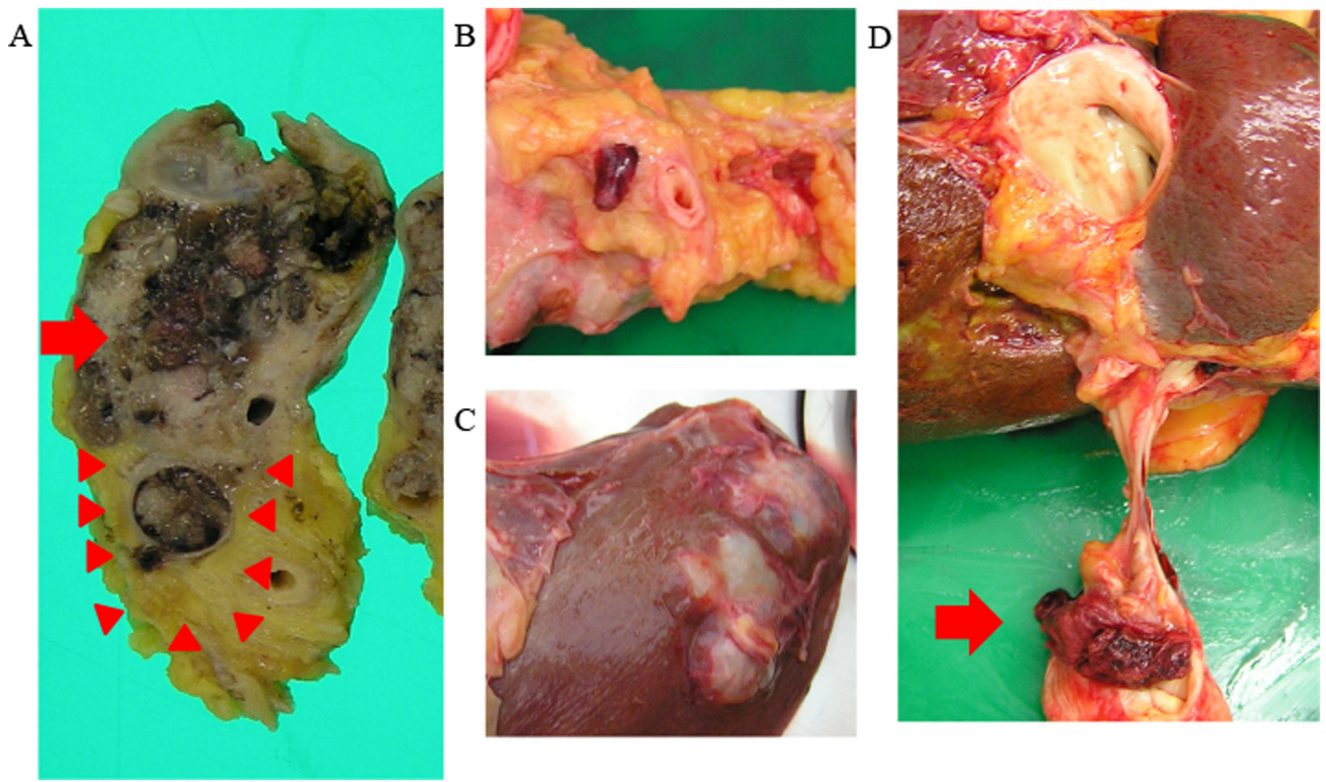

Figure 4. Macroscopic findings. (A) The pancreatic head tumor (arrow) invaded the portal vein (arrowheads). (B) SMV tumor thrombosis. (C) Metastatic liver lesion. (D) The tumor thrombus extended from the SMV to the hilar portal vein (arrow). SMV, superior mesenteric vein.

tumor thrombosis. Second, the pancreatic tumor metastasized to the liver via the portal vein, retained the IPMC structure and produced mucus. Currently, invasive IPMN is considered to have a better prognosis compared with conventional PDAC $(7,8)$. Ueda et al $(9)$ reported the case of a female patient with IPMN with metastases to the lymph nodes, liver and lung, who achieved long-term survival (5 years and 2 months), as she responded to chemotherapy following pancreaticoduodenectomy. However, in the present case, the patient's survival duration after diagnosis was very short due to the widespread local invasion, distant metastases and lack of anticancer treatment, such as systemic chemotherapy or radiotherapy.
IPMN and other cystic pancreatic tumors may be accurately and timely diagnosed due to the advances in imaging techniques, including MRI and ERCP (10). On occasion, these tumors are incidentally found on abdominal CT in asymptomatic patients. Other patients may present with variable symptoms, such as abdominal pain, obstructive jaundice and vomiting, as in the present case. The progression of obstructive jaundice with IPMN has been previously reported, as mucus produced from IPMN may obstruct the common bile duct (11). However, in the present case, the common bile duct was directly invaded and obstructed by the tumor. 

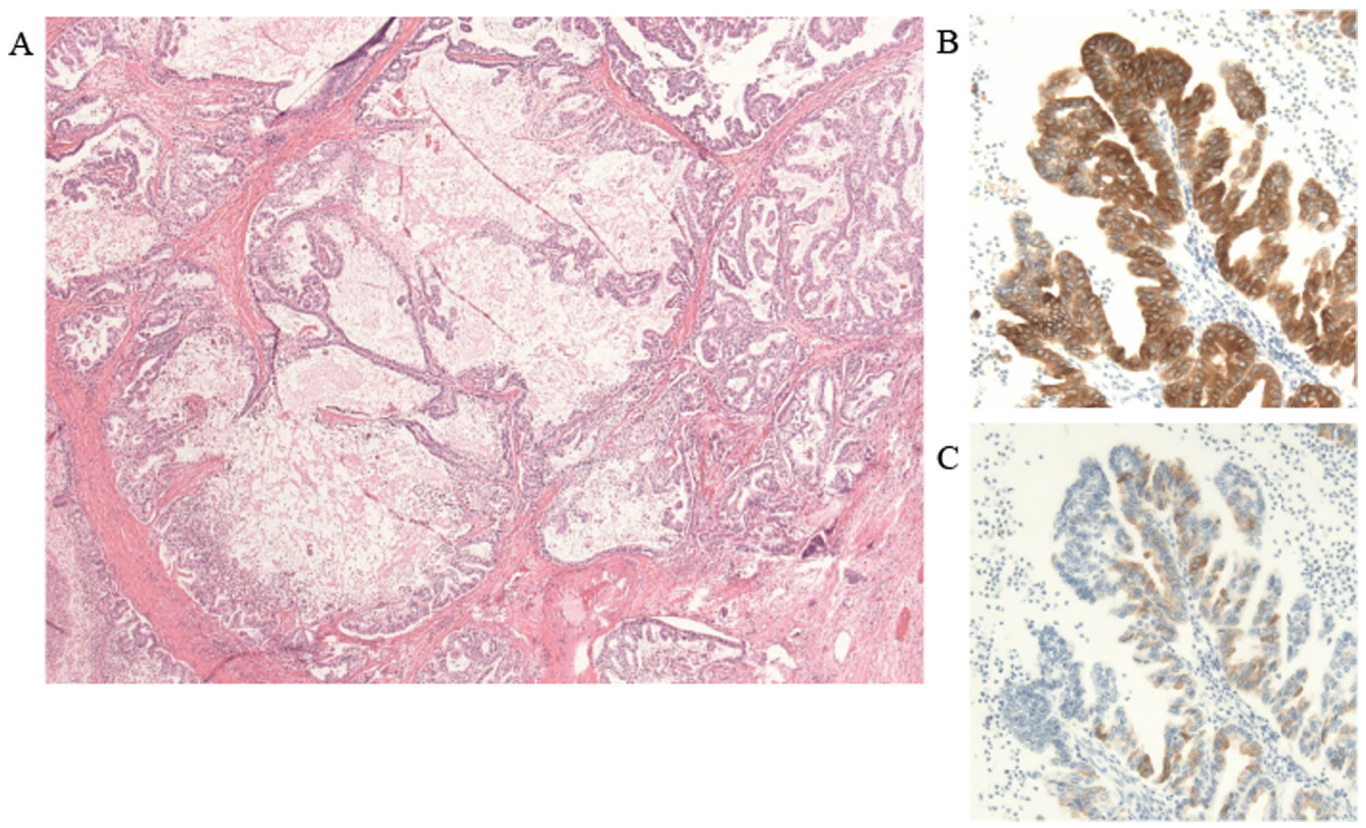

Figure 5. Microscopic findings of the pancreatic head lesion. (A) The tumor displayed a papillary structure consisting of small tumor cells. The cysts contained mucus (hematoxylin and eosin staining; magnification, x40). (B) Positive MUC1 staining (magnification, x100). (C) Slightly positive MUC5AC staining (magnification, x100). MUC, mucin core protein.

A

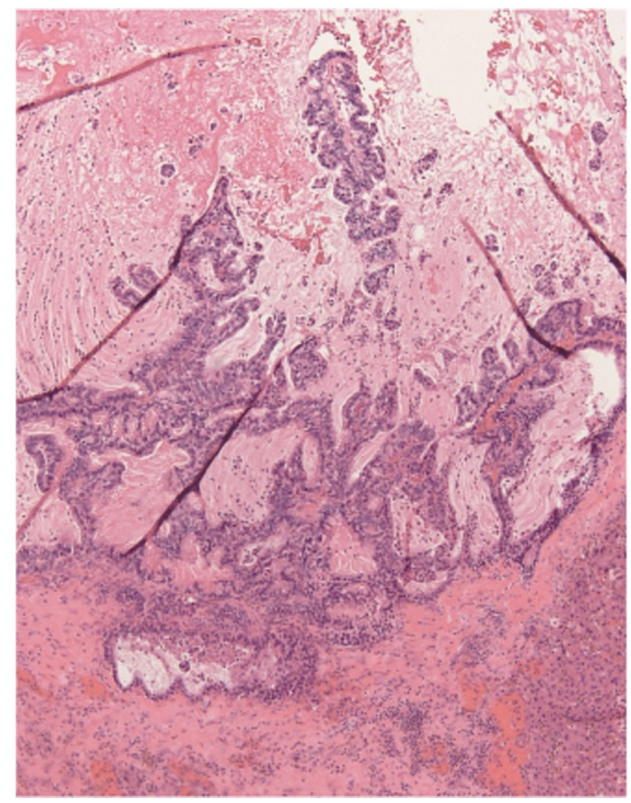

B

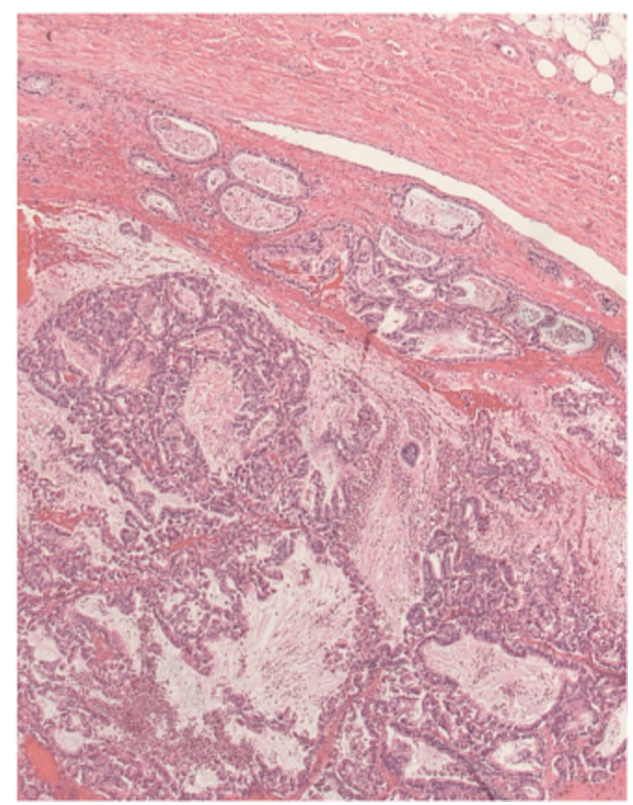

Figure 6. Microscopic findings of the liver metastatic lesion (hematoxylin and eosin staining; magnification, x40). (A) The polycystic liver tumors had the same structure as the pancreatic tumors on pathological examination. The polycystic lesions displayed a papillary arrangement with mucus pooling. (B) The structure of the superior mesenteric vein tumor thrombus was identical to that of the primary pancreatic tumor.

Serum CA19-9 level is a well-known predictor of PDAC and poor survival. Elevated serum CA19-9 levels were found to be associated with mixed invasive IPMC, which is consistent with several previous reports $(12,13)$, and this finding may indicate that invasive IPMC shares similar characteristics with PDAC. Hirono et al (14) evaluated the diagnostic value of the factors associated with invasive IPMC identified in their study, and branch-duct IPMN with elevated serum CA19-9 levels was associated with invasive IPMC, with a sensitivity of $86.4 \%$, specificity of $96.6 \%$, positive predictive value of $86.4 \%$, negative predictive value of $96.6 \%$ and accuracy of $94.5 \%$.

IPMNs are classified into four subtypes (intestinal, pancreatobiliary, gastric and oncocytic) based on their histomorphology and mucin phenotype (15). The subtype of the present case was pancreatobiliary, as determined based on the positive expression of MUC1 and MUC5AC and negative expression of MUC2. The pancreatobiliary type is the most malignant among the four subtypes (5). Moreover, the main-duct or mixed-type morphological classifications of 
IPMN are associated with a higher risk of evolving into invasive IPMC compared with the branch-duct type (16). Mixed-type IPMN includes various histological types. Masuda et al (17) previously reported that mixed IPMN with positive MUC2 expression had a significantly higher prevalence of high-grade dysplasia and invasive IPMC compared with MUC2-negative IPMN. However, the tumor in the present case was classified as invasive IPMC, despite the negative MUC2 expression. It was previously reported that the mixed type of IPMN rarely includes the pancreatobiliary type (17). The pancreatobiliary type is MUC2-negative, as are the gastric and oncocytic types. Thus, this contradiction regarding whether mixed-type IPMN is invasive may be attributed to selection bias in the report.

It was reported that $19-45 \%$ of IPMNs are accompanied by invasive carcinomas (8). However, the presence of portal vein invasion was significantly lower among patients with invasive IPMC compared with those with PDAC (8). Invasive IPMCs with portal vein invasion and tumor thrombosis, such as in the present case, have rarely been reported $(16,18)$. Moreover, our patient had a long tumor thrombus and liver metastases. When an abdominal contrast-enhanced CT was first performed, the tumor thrombus was only $10 \mathrm{~mm}$. However, when the autopsy was performed after 90 days, the tumor thrombus had grown and extended from the SMV to the hilar portal vein. The rapid growth of the portal thrombosis indicated a highly malignant tumor. In addition, invasion into the vasculature is a significant predictor of poor outcome (19). Moreover, in the present case, the structure of the polycystic liver tumors was identical to that of the pancreatic tumors on pathological examination. The SMV tumor thrombosis metastasized to the liver, maintaining its polycystic structure and mucus production. As the pancreatic head tumor had not metastasized to the regional lymph nodes, it was inferred that part of the SMV tumor thrombus, with a polycystic structure and mucus content, was detached and reached the liver via the portal vein.

While the patient and his family opted for best supportive care in the present case, several therapies for invasive IPMC have been reported. Pancreatectomy with lymph node dissection are recommended for invasive IPMC and PDAC, if the tumor is resectable (20), whereas the efficacy of postoperative adjuvant therapy for invasive IPMC remains controversial. McMillan et al (21) reported that postoperative adjuvant therapy may improve the survival of patients with advanced-stage invasive IPMC or lymph node metastases. However, another study reported that postoperative adjuvant therapy does not affect the survival of patients with invasive IPMC (22). Moreover, it is unclear whether systemic chemotherapy improves the survival of patients with unresectable invasive IPMN. Chemoradiotherapy has been demonstrated to prolong the survival of patients with PDAC (23). Regarding chemotherapy for PDAC, gemcitabine or S-1 (tegafur, gimeracil, oteracil potassium) as systemic chemotherapy for unresectable invasive IPMC is clinically considered (24).

To date, there have been reports of IPMC with portal invasion or liver metastasis $(25,26)$. However, to the best of our knowledge, this is the first report of a IPMC metastasizing to the liver while maintaining its IPMC structure.

As regards the limitations of the present study, the expression of proliferating cell nuclear antigen, tumor protein $\mathrm{p} 53$, vascular endothelial growth factor and the KRAS mutation status were not determined due to health insurance-related restrictions. From an academic perspective, however, immunohistochemical examination must be performed to evaluate the status of carcinogenesis.

In conclusion, we herein present our experience with an autopsy case of IPMC accompanied by a long tumor thrombus in the portal vein and multifocal liver metastasis that maintained the structure of the primary pancreatic head tumor.

\section{Acknowledgements}

Not applicable.

\section{Funding}

No funding was received.

\section{Availability of data and materials}

All data generated or analyzed during this study are included in this published article.

\section{Authors' contributions}

NM wrote the report. NM and AD were the attending physicians for the patient. NM, AD, HU, SA, KM and KY conducted the ERCP. MT made the pathological diagnosis. AD supervised the report. All the authors have read and approved the final version of the manuscript.

\section{Ethics approval and consent to participate}

Not applicable.

\section{Patient consent for publication}

The family of the patient consented to the publication of the case details and associated images.

\section{Competing interests}

The authors declare that they have no competing interests.

\section{References}

1. Tanaka M, Fernández-Del Castillo C, Kamisawa T, Jang JY, Levy P, Ohtsuka T, Salvia R, Shimizu Y, Tada M and Wolfgang CL: Revision of international consensus Fukuoka guidelines for the management of IPMN of the pancreas. Pancreatology 17: 738-753, 2017.

2. Adsay V, Mino-Kenudson M, Furukawa T, Basturk O,Zamboni G, Marchegiani G, Bassi C, Salvia R, Malleo G, Paiella S, et al: Pathologic evaluation and reporting of intraductal papillary mucinous neoplasms of the pancreas and other tumoral intraepithelial neoplasms of pancreatobiliary tract: Recommendations of verona consensus meeting. Ann Surg 263: 162-177, 2016.

3. Adsay NV, Merati K, Basturk O, Iacobuzio-Donahue C, Levi E, Cheng JD, Sarkar FH, Hruban RH and Klimstra DS: Pathologically and biologically distinct types of epithelium in intraductal papillary mucinous neoplasms: Delineation of an 'intestinal' pathway of carcinogenesis in the pancreas. Am J Surg Pathol 28: 839-848, 2004. 
4. Aronsson L, Andersson R and Ansari D: Intraductal papillary mucinous neoplasm of the pancreas-epidemiology, risk factors, diagnosis, and management. Scand J Gastroenterol 52: 803-815, 2017.

5. Kim J, Jang KT, Mo Park S, Lim SW, Kim JH, Lee KH, Lee JK, Heo JS, Choi SH, Choi DW, et al: Prognostic relevance of pathologic subtypes and minimal invasion in intraductal papillary mucinous neoplasms of the pancreas. Tumour Biol 32: 535-542, 2011.

6. James D. Brierley and Marry K: Gospodarowicz, Christian Wittekind. TMN Classification of Malignant Tumors, 8th ed. New York, Wiley-Blackwell, 2016.

7. Sohn TA, Yeo CJ, Cameron JL, Hruban RH, Fukushima N, Campbell KA and Lillemoe KD: Intraductal papillary mucinous neoplasms of the pancreas an updated experience. Ann Surg 239: 788-797 discussion 797-9, 2004

8. Murakami Y, Uemura K, Sudo T, Hayashidani Y, Hashimoto Y, Nakashima A and Sueda T: Invasive intraductal papillary-mucinous neoplasm of the pancreas: Comparison with pancreatic ductal adenocarcinoma. J Surg Oncol 100: 13-18, 2009.

9. Ueda N, Kaida D, Tomita Y, Ohnishi T, Funaki H, Fujita H, Kinami S, Nakano Y and Kosaka T: A patient with lnvasive carcinoma derived from IPMN who achieved long-term survival despite lymph node metastasis. Gan To Kagaku Ryoho 41: 2202-2204, 2014 (In Japanese).

10. Asskan G, Bağci P, Memiş B and Baștürk O: Intraductal neoplasms of the pancreas: An update. Turk Patoloji Derg 33: 87-102, 2017.

11. Patel A, Lambiase L, Decarli A and Fazel A: Management of the mucin filled bile duct. A complication of intraductal papillary mucinous tumor of the pancreas. JOP 6: 255-259, 2005.

12. Fritz S, Hackert T, Hinz U, Hartwig W, Büchler MW and Werner J: Role of serum carbohydrate antigen 19-9 and carcinoembryonic antigen in distinguishing between benign and invasive intraductal papillary mucinous neoplasm of the pancreas. $\mathrm{Br}$ J Surg 98: 104-110, 2011.

13. Kim JR, Jang JY, Kang MJ, Park T, Lee SY, Jung W, Chang J, Shin Y, Han Y and Kim SW: Clinical implication of serum carcinoembryonic antigen and carbohydrate antigen 19-9 for the prediction of malignancy in intraductal papillary mucinous neoplasm of pancreas. J Hepatobiliary Pancreat Sci 22: 699-707, 2015.

14. Hirono S, Kawai M, Okada KI, Miyazawa M, Shimizu A, Kitahata Y, Ueno M, Yanagisawa A and Yamaue H: Factors associated with invasive intraductal papillary mucinous carcinoma of the pancreas. JAMA Surg 152: e165054, 2017

15. Furukawa T: Subtyping of IPMN. Methods Mol Biol 1882: 1-8, 2019.

16. Castellano-Megías VM, Andrés CI, López-Alonso G and Colina-Ruizdelgado F: Pathological features and diagnosis of intraductal papillary mucinous neoplasm of the pancreas. World J Gastrointest Oncol 6: 311-324, 2014.
17. Masuda A, Arisaka Y, Hara S, Matsumoto I, Takenaka M, Sakai A, Shiomi H, Matsuki N, Sugimoto M, Fujita T, et al: MUC2 expression and prevalence of high-grade dysplasia and invasive carcinoma in mixed-type intraductal papillary mucinous neoplasm of the pancreas. Pancreatology 13: 583-588, 2013.

18. Tomimaru Y, Ishikawa O, Ohigashi H, Eguchi H, Yamada T, Sasaki Y, Kishi K, Takachi K, Noura S, Miyashiro I, et al: Intraductal papillary-mucinous carcinoma of the pancreas with tumor thrombus in the portal vein: A report of two cases. Hepatogastroenterology 54: 1585-1588, 2007.

19. D'Angelica M, Brennan MF, Suriawinata AA, Klimstra D and Conlon KC: Intraductal papillary mucinous neoplasms of the pancreas: An analysis of clinicopathologic features and outcome. Ann Surg 239: 400-408, 2004.

20. Hirono S and Yamaue H: Surgical strategy for intraductal papillary mucinous neoplasms of the pancreas. Surg today 50: 50-55, 2020.

21. McMillan MT, Lewis RS, Drebin JA, Teitelbaum UR, Lee MK, Roses RE, Fraker DL and Vollmer CM: The efficacy of adjuvant therapy for pancreatic invasive intraductal papillary mucinous neoplasm (IPMN). Cancer 122: 521-533, 2016.

22. Hirono S, Shimizu Y, Ohtsuka T, Kin T, Hara K, Kanno A, Koshita S, Hanada K, Kitano M, Inoue H, et al: Recurrence patterns after surgical resection of intraductal papillary mucinous neoplasm (IPMN) of the pancreas; a multicenter, retrospective study of 1074 IPMN patients by the Japan Pancreas Society. J Gastroentrol 55: 86-99, 2020.

23. Ochiai T, Igari K, Furuyama T, Ito H, Mitsunori Y, Aihara A, Kumagai Y, Iida M, Odajima H, Tanaka S, et al: Favorable response after Gemcitabine-Radiotherapy for invasive pancreatic intraductal papillary mucinous neoplasm: A case report. Int Surg 98: 340-345, 2013.

24. Kameyama S, Motonari H, Ishimine T and Isa T: Successful treatment with conversion surgery following chemoradiotherapy for unresectable invasive intraductal papillary mucinous neoplasm. Clin J Gastroenterol 13: 579-584, 2020

25. Marsoner K, Haybaeck J, Csengeri D, Waha JE, Schagerl J, Langeder R, Mischinger HJ and Kornprat P: Pancreatic resection for intraductal papillary mucinous neoplasm- a thirteen-year single center experience. BMC Cancer 16: 844, 2016.

26. Matsuda Y,Hagio M, Naito Z and Ishiwata T: Clinicopathological features of 30 autopsy cases of pancreatic carcinoma. J Nippon Med Sch 79: 459-467, 2012.

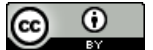

This work is licensed under a Creative Commons Attribution 4.0 International (CC BY 4.0) License. 